\title{
COUPLING OF A CMOS OPTICAL SENSOR TO A MICROMACHINED DEFORMABLE MIRROR WITH AN ADALINE NEURAL METHOD
}

\author{
Davies W. de Lima Monteiro, Antônio I. Ferreira Jr., Frederico B. Teixeira, \\ João Guilherme M. Melo and Gleb Vdovin* \\ OptMA $^{\text {lab }}$, Department of Electrical Engineering, UFMG, Brazil \\ *EI Lab.- Delft University of Technology/ Flexible Optical B.V., The Netherlands \\ Av. Antônio Carlos , 6627 - 31270-010 Belo Horizonte-MG, Brazil, davies@ufmg.br
}

\begin{abstract}
We report on the preliminary results of an Adaline neural method for the coupling of a custom CMOS wavefront sensor to a micromachined adaptive mirror. The algorithm does not rely on a fixed basis matrix -as opposed to traditional methods-, offers excellent immunity to round-off errors and admits real-time input adaptability to speed up computations.
\end{abstract}

\section{INTRODUCTION}

There has been an increasing demand for precision in the detection and correction of aberrations in optical systems, so that the maximization of quality parameters (e.g.: sharpness, power, uniformity) is achieved. These systems encompass telescopes, microscopes, the human eye and many others in industry, science and medicine.

The whole detection and correction process is based on the concept of a wavefront, whose profile is affected by its propagation media (e.g.: cornea, atmospheric layers, lenses, etc). Detection and correction of wavefront aberrations, and consequently of the chief optical system, are achieved by a secondary optical system consisting basically of a wavefront sensor and an adaptive mirror. The coupling of these elements is usually performed by a control algorithm based on matrix algebra, where a fixed and necessarily invertible basis matrix stores the sensor responses to basic mirror modes. In this paper we present the preliminary results of a simple Adaline neural method both for the diagnosis of a wavefront aberration and for the control algorithm for an adaptive optical system (1).

\section{WAVEFRONT RECONSTRUCTION PRINCIPLES}

A wavefront is a hypothetical surface representing the points with equal phase in a beam. A flat wavefront is therefore a plane perpendicular to the direction of propagation of a collimated and monochromatic beam and limited by the beam diameter. If this flat wavefront traverses a homogeneous and isotropic transparent optical component with a given profile, for instance the human cornea, it adopts its shape with amplitude multiplied by the factor $(n-1)$, where $n$ is the index of refraction of the component.

In the Hartmann wavefront sensing method, the probing laser beam impinges on an opaque mask with a number of sub-apertures. The beam is sampled as $N$ sub-beams, 
which propagate a small distance (usually from 1 to $10 \mathrm{~cm}$ ) towards an observation screen. Assuming Fresnel diffraction does not impart too much power divergence from mask to screen and that the wavefront is flat, then, the resulting spots will unmistakenly reproduce on the screen the sub-aperture grid present on the mask. Distortions on the wavefront result in the departure of the light spots from the initial grid. Recording the $x$ and $y$ displacements of the spots from the $(i, j)$ grid points, one can geometrically calculate the respective $(i, j)$ wavefront slopes and therefore reconstruct the initial wavefront.

The reconstruction of a wavefront from a set of displacements basically means solving a system of linear equations, which is conveniently tackled by a matrix equation in the form $\boldsymbol{s}=\mathbf{B} \boldsymbol{c}$. In modal reconstruction, $\mathbf{B}$ is an $L x 2 N$ basis matrix that contains the $x$ and $y$ displacements, at the $N$ sub-aperture grid points, corresponding to $L$ linearly independent optical functions (tip, tilt, defocus, astigmatism, spherical aberration, coma, trefoil, etc), which are usually Zernike polynomials (2). Vector $\boldsymbol{s}$ contains the $N \quad x$ and $y$ displacements for an arbitrary wavefront, and $\boldsymbol{c}$ is a vector of L coefficients (weights to the optical functions).

In practice, since $\boldsymbol{s}$ is usually dimensionally larger than $\boldsymbol{c}$, the system is overdetermined and the conventional least-squares method works to minimize the error between the left and right-hand sides of the above matrix equation. The convergence of this method requires that matrix $\mathbf{B}$ be neither singular nor nearly singular, which might be undermined by round-off errors during computations. Although robust concurrent methods are available (iterative methods and SVD), they still rely on a fixed basis matrix and do not offer the real-time adaptability an Adaline neural method does.

A test wavefront $\Xi_{W}$ can be represented on a native basis, for instance, Zernike polynomials $Z_{i}()$ :

$$
\Xi_{W}=\sum_{i=0}^{\infty} C_{i} Z_{i}(\rho, \theta)
$$

The sensor signals, associated with the test wavefront, yield a reconstructed wavefront $\Xi_{R}$ that can be decomposed on a reconstruction basis with $L$ functions and their respective $\lambda_{i}$ coefficients, as in Equation [2]. The reconstruction order $L$ is dictated mostly by the sensor geometry and noise.

$$
\Xi_{R}=\sum_{i=0}^{L} \lambda_{i} Z_{i}(\rho, \theta)+\lambda_{L+1} \cdot(-1)
$$

In some cases, the weighted sum truncated to the first $L$ terms might suffice for the wavefront reconstruction, as in ophthalmology where wavefront aberrations in the human eye have been observed to be substantial only to the first 14th Zernike orders.

\section{WAVEFRONT DIAGNOSIS WITH AN ADALINE NEURAL METHOD}

For the diagnosis of test wavefronts, the algorithm receives as inputs the reconstruction basis, consisting of $L=14$ Zernike functions. It outputs a weighted sum $\Xi_{R}$ that is 
compared to the test wavefront $\Xi_{W}$ at each grid coordinate ( $\rho$ and $\theta$ ). Then, the meansquare error (mse) of the resulting residual matrix $\Xi_{W}-\Xi_{R}$ serves as feedback to the neural net. Each internal iteration (epoch) adjusts the 14 nodal weights $\left(\lambda_{i}\right)$ towards mse minimization. The internal algorithm step, ruling the gradient decrease on a hyperparabolic surface, is set consecutively as $\eta=0.1$ and $\eta=0.01$. The stop parameters are mse $\leq 1 e-6$ and epochs $s_{\max }=1000$. Figure 1 illustrates the Adaline topology. The ultimate weights indicate the best coefficients $\left(\lambda_{i}\right)$ to the respective Zernike terms in order to reconstruct the test wavefront. Data permutation in this algorithm prevents that it stacks at pseudo-minimum error levels.

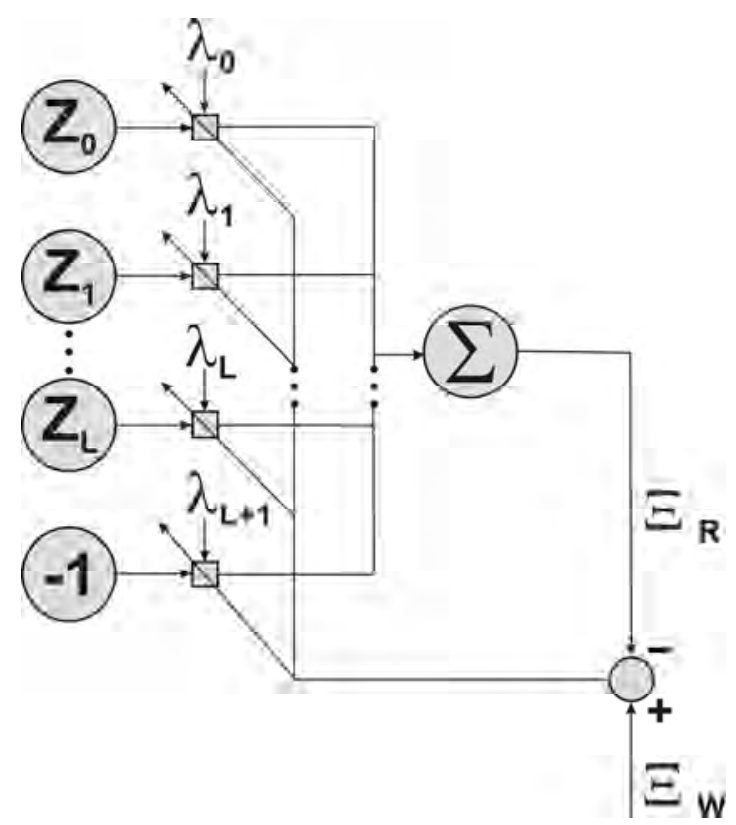

Figure 1: Adaline neural method topology.

We executed the algorithm for six different test wavefronts, represented as a weighted sum to the $k^{\text {th }}$ order, where the conditions are described below. The purpose of each test is indicated within parentheses.

test 1 - $k=L, C_{i}=1$ for $i=0, \ldots, 13$ (direct matching);

test 2 - $k<L, C_{0}=1, C_{i}=0$ for $i \neq 0$ (residual cross-coupling);

test $3-k<L, C_{i}=1$ for $i=0,2,4,5$ and $C_{i}=0.1$ for $i \neq 0,2,4,5$ (classification ability and residual error for a highly asymmetric wavefront);

test 4 - $k>L, C_{i}=1$ for $i<14$ and $C_{i}=0.1$ for $i=14$ (aliasing with one additional Zernike term);

test $5-k>L, C_{i}=1$ for $i<14$ and $C_{i}=0.1$ for $i=14, \ldots, 16$ (aliasing with three additional Zernike terms);

test $6-k>L, C_{i}=1$ for $i<14$ and $C_{i}=0.1$ for $i=14, \ldots, 19$ (aliasing with six additional Zernike terms).

For each test we registered the number of epochs and respective mse achieved. The rms wavefront error is the normalized error to the maximum amplitude. The results are presented in Table 1. 
Table 1: Test results with the Adaline neural method

\begin{tabular}{|c|c|c|c|c|c|}
\hline \multirow{2}{*}{$\#$} & \multirow{2}{*}{ Epoch } & $\eta=0.1$ & $\eta=0.01$ & $\eta=0.1$ & $\eta=0.01$ \\
\cline { 3 - 6 } & & $m s e$ & $r m s$ & $m s e$ & $r m s$ \\
\hline 1 & 1 & $2.6 \mathrm{e}-14$ & $1.2 \mathrm{e}-7$ & $6.2 \mathrm{e}-11$ & $3.7 \mathrm{e}-6$ \\
\hline 2 & 1 & $2.3 \mathrm{e}-14$ & $1.3 \mathrm{e}-7$ & $8.2 \mathrm{e}-7$ & $9.0 \mathrm{e}-4$ \\
\hline 3 & 1 & $9.5 \mathrm{e}-14$ & $1.7 \mathrm{e}-7$ & $6.6 \mathrm{e}-7$ & $7.8 \mathrm{e}-4$ \\
\hline \multirow{2}{*}{4} & 1 & $1.1 \mathrm{e}-3$ & $3.5 \mathrm{e}-2$ & $9.0 \mathrm{e}-4$ & $3.1 \mathrm{e}-2$ \\
\cline { 2 - 6 } & $1 \mathrm{e} 3$ & $8.4 \mathrm{e}-4$ & $3.2 \mathrm{e}-2$ & $8.6 \mathrm{e}-4$ & $2.9 \mathrm{e}-2$ \\
\hline \multirow{2}{*}{5} & 1 & $3.8 \mathrm{e}-3$ & $5.9 \mathrm{e}-2$ & $2.2 \mathrm{e}-3$ & $4.9 \mathrm{e}-2$ \\
\cline { 2 - 6 } & $1 \mathrm{e} 3$ & $2.8 \mathrm{e}-3$ & $6.0 \mathrm{e}-2$ & $2.3 \mathrm{e}-3$ & $4.7 \mathrm{e}-2$ \\
\hline \multirow{2}{*}{6} & 1 & $6.4 \mathrm{e}-3$ & $7.6 \mathrm{e}-2$ & $4.5 \mathrm{e}-3$ & $6.3 \mathrm{e}-2$ \\
\cline { 2 - 6 } & $1 \mathrm{e} 3$ & $6.6 \mathrm{e}-3$ & $7.5 \mathrm{e}-2$ & $4.3 \mathrm{e}-3$ & $6.2 \mathrm{e}-2$ \\
\hline
\end{tabular}

Tests 1 through 3 indicate that a single epoch is sufficient to achieve minimum error provided that the test wavefront can be completely described by the set of reconstruction functions $(k \leq L)$.

Tests 4 through 6 reveal that higher spatial frequency terms $(k>L)$ must be somehow described by a restricted range of Zernike terms, resulting in an increase in aliasing and in a worse minimum error as more high-order terms are added. The aliasing error decreases for $\eta=0.01$ and remains practically unaltered regardless the number of epochs used. This derives from the fact that the algorithm was set to operate with a fixed number of inputs throughout the tests. The algorithm can be easily extended to include dynamic adaptability, where the number of Zernike reconstruction functions $L$ can be changed between epochs depending on the mse convergence rate.

\section{THE ADAPTIVE OPTICAL SYSTEM}

The Adaptive Optical (AO) system was set up as shown in Figure 2. The laser beam (12mm diameter, He-Ne, $\lambda=632.8 \mathrm{~nm}$ ) traverses the aberration plane (AB), impinges on the mirror and is reflected towards a beam-splitter that divides the beam between the custom CMOS wavefront sensor and a conventional CCD-based wavefront sensor. The planes of the mirror and the sensors are optically conjugated to the aberration plane by means of two relay-lens systems.

The mirror is a 15-mm micromachined membrane deformable mirror (MMDM), Flexible Optical B.V., with 37 channels and electrostatic actuation. The membrane is kept at a bias position by maintaining an offset voltage of $\sim 160 \mathrm{~V}$ at all actuators with respect to the grounded membrane. The channel voltage can reach up to $250 \mathrm{~V}$ and the maximum mirror stroke is $8 \mu \mathrm{m}$. 


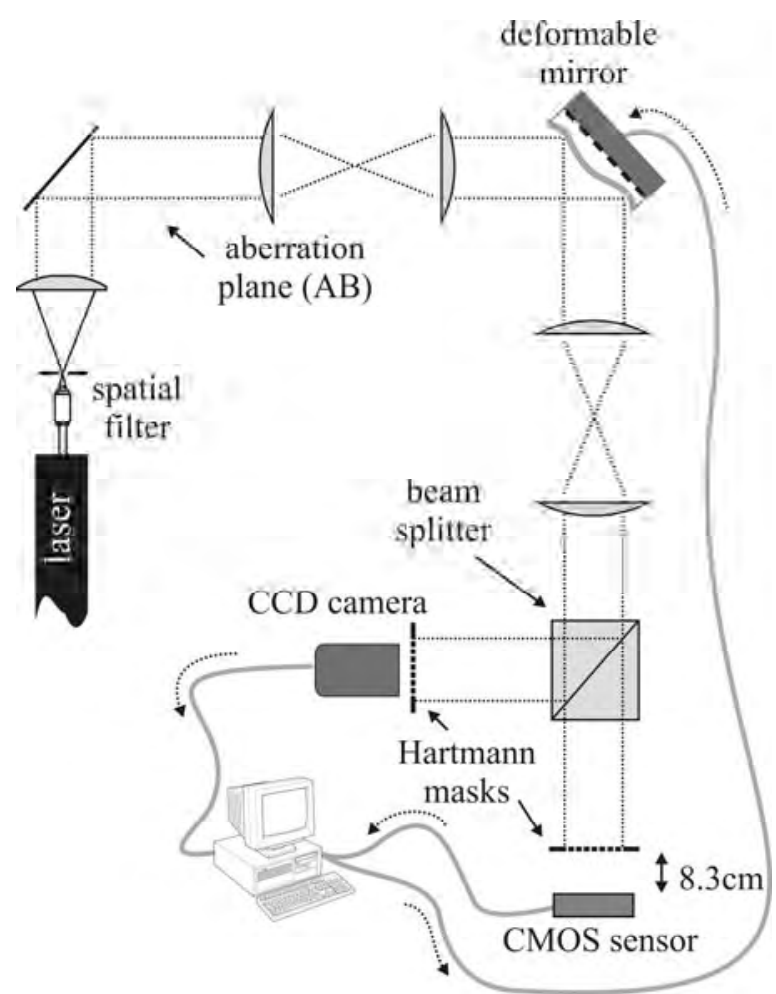

Figure 2: Adaptive Optical system.

The custom wavefront sensor consists of an opaque mask (Hartmann mask) with sixteen $450 \mu \mathrm{m}$ circular sub-apertures laid on a regular square array with $1000 \mu \mathrm{m}$ pitch. The mask is positioned at $8.3 \mathrm{~cm}$ away from a CMOS chip with a corresponding array of quad-cells (QCs) $600 \mu \mathrm{m}$ wide (3). Each pixel in a QC is passive and based on a double-junction structure $\left(\mathrm{p}^{+} / \mathrm{n}\right.$-well $/ \mathrm{p}$-epi). The spot intensity profile, centered on the QC, is shown in along with the QC response to spot displacements over its area. The 64 pixels are selected by an internal multiplexer and their signals are prompted at four parallel output lines. The custom chip outperforms an off-the-shelf camera in operational speed because it delivers signals directly proportional do the $x$ and $y$ displacements, circumventing the need for image processing.

The quad-cell response is clearly non-linear and can be closely fit with a sigmoidal curve. Although it is not detrimental to wavefront accuracy, upon calibration, linearization of this curve could speed up the numerical routines that relate the sensed signal to true displacements.

The data-acquisition card is a custom ethernet board, Lynx Ltda., from which we used 4 locked digital outputs and 4 analog inputs with 12-bit resolution. Sensor signals are communicated to the computer (Pentium IV, 2.4GHz) via a UDP protocol. The sensor was readout at a frequency of $1 \mathrm{kHz}$.

The conventional CCD-based sensor was used to monitor the test aberrations statically with FrontSurfer, a wavefront analysis software distributed by Flexible Optical B.V. The camera sensor is a low-light monochromatic CCD that captures the image of a Hartmann mask with a $6^{\text {th }}$-order hexagonal array with 91 sub-apertures, where each is $350 \mu \mathrm{m}$ in diameter. The mask is placed at $1.3 \mathrm{~cm}$ from the camera. 


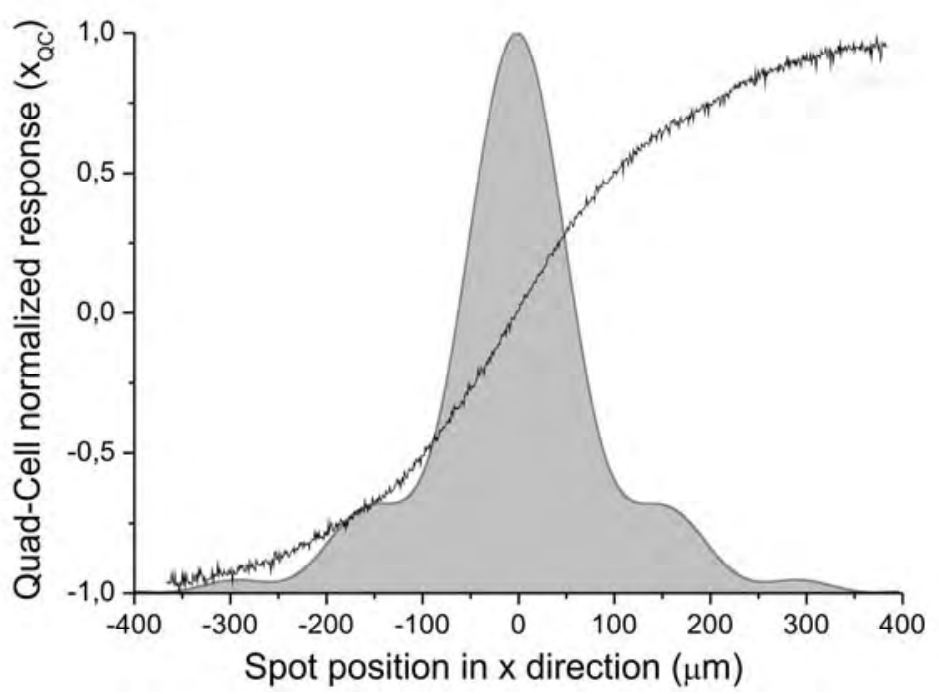

Figure 3: Spot intensity profile at $8.3 \mathrm{~cm}$ from the mask superimposed on the quad-cell response to displacements in the $x$ direction.

\section{CONTROL OF THE DEFORMABLE MIRROR}

A mirror mode, or influence function, is the shape the membrane assumes when an incremental voltage step is applied to a single mirror electrode (actuator). For the mirror we used, there are thus 37 modes, each imparting a characteristic set of spot displacements on the CMOS wavefront sensor. We can consider the wavefront reflected from a biased membrane to be the reference wavefront, itself responsible for a set of $x$ and $y$ displacements marking reference positions.

To cancel out any aberration introduced at plane $A B$, the mirror membrane must assume a shape that reproduces that distortion with twice as low amplitude to account for a phase path twice as large upon reflection (4). An aberrated wavefront reflected from a counterbalanced membrane incidently means the center of gravity of every light spot is driven back to its reference position. Therefore, from the control point of view, restoration can be achieved by identifying mirror shapes that minimize spot departures from their original grid.

The Adaline neural method used for Zernike terms has been modified to attend to this system. The topology remains basically the same, but the input reconstruction functions have been substituted with the sensor responses to the 37 mirror modes $M_{i}$. Each mode corresponds to a vector with 32 elements representing sixteen $x$ and $y$ displacements. The algorithm delivers an output vector $D$, as a weighted sum of the basis vectors, in an attempt to minimize the error to a sample vector $D_{d}$ associated with the aberrated wavefront $\Xi_{W}$. The weights $w_{i}(\mathrm{i}=0, \ldots, 36)$ are related to the voltages to be applied to the mirror actuators. Refer to Figure 4 for the adapted Adaline topology. 


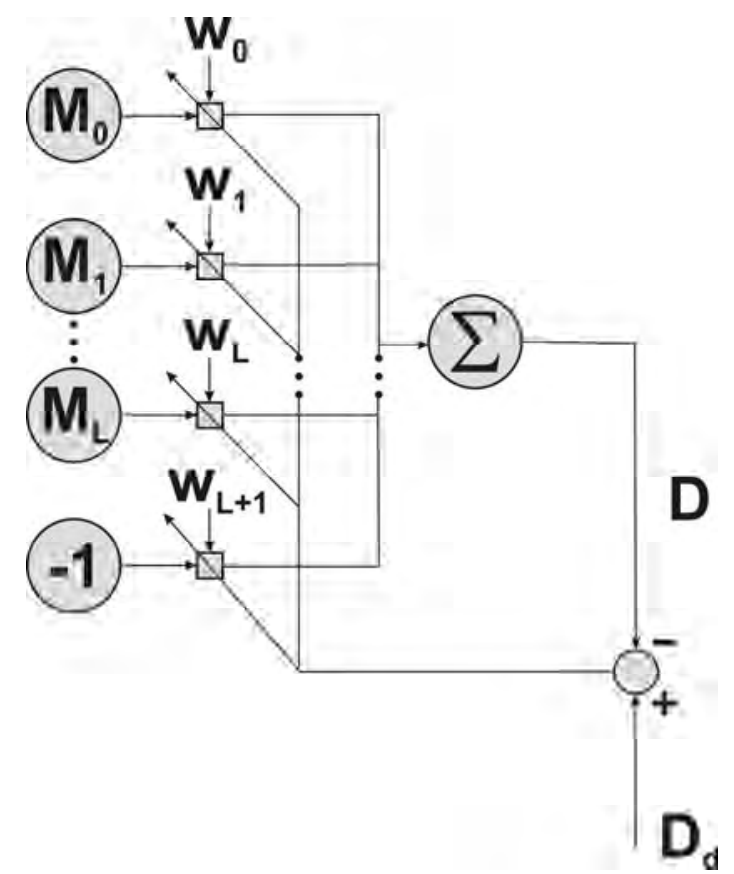

Figure 4: Adaline topology for an Adaptive Optical system

\section{PERFORMANCE OF THE ADALINE ALGORITHM}

We introduced centered defocus with amplitude of $36 \mu \mathrm{m}(>55 \lambda)$ as the aberration. This value is far beyond the working stroke of the mirror membrane and is only intended to evaluate the convergence ability of the algorithm. This aberration exploits the spatial dynamic range of the quad-cells because it forces the outer spots to the edge of their respective QCs. The Adaline parameter mse indicates how close the solution is to the reference grid as a function of the number of epochs. The positional resolution $r_{m} s_{P}$ normalized to displacements from -1 to 1 is related to mse as indicated in Equation [3].

$$
r m s_{P}=\frac{2 N \cdot \sqrt{m s e}}{(2 N-1)^{2} \cdot(M+2)},
$$

where $N$ is the number of quad-cells in the wavefront sensor and $\mathrm{M}$ is the number of mirror modes. Figure 5 presents how the normalized positional error evolves with the number of epochs. For all epochs the error is well under $1 \%$.

Next we introduced a milder aberration with amplitude $\lambda / 2$ (Figure 6) to check the algorithm convergence.

When the neural algorithm starts with unity weights it takes a single epoch to reach an acceptable solution, i.e. a wavefront match better than $\lambda / 50$. After $\sim 80$ s and $1.5 \mathrm{M}$ epochs the mse reaches $0.00017\left(r m s_{p}<3 \mathrm{e}^{-3}\right.$, wavefront match better than $\left.\lambda / 5000\right)$. 


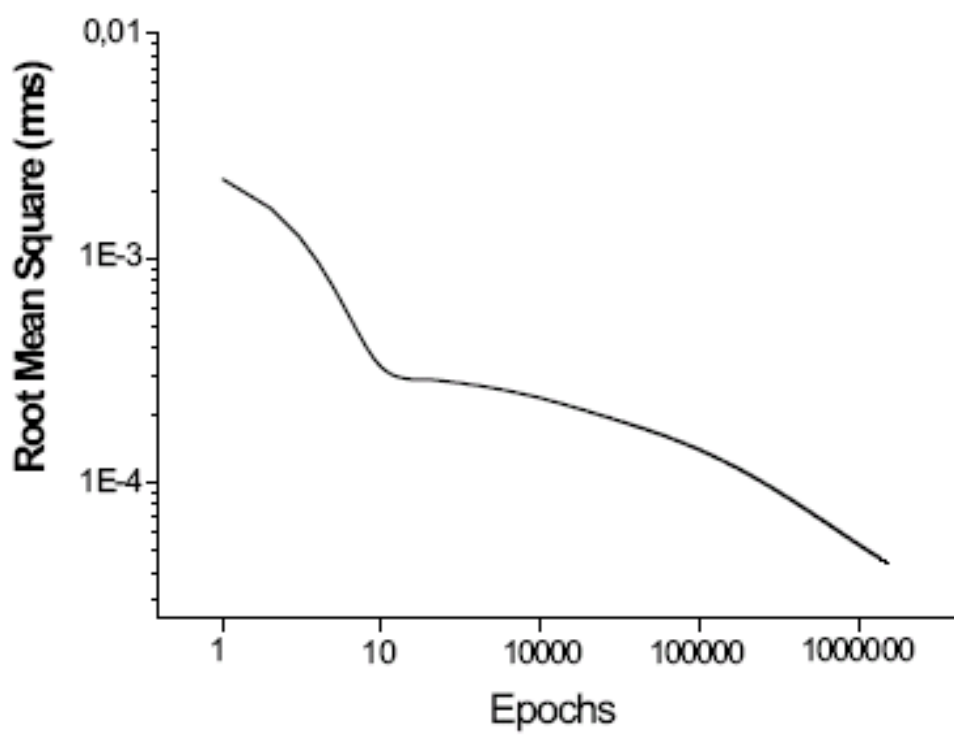

Figure 5: Mean-square error ( $m s e$ ) as a function of the number of epochs for an extreme situation in which the amplitude of the wavefront aberration imparts maximum detectable spot displacements.

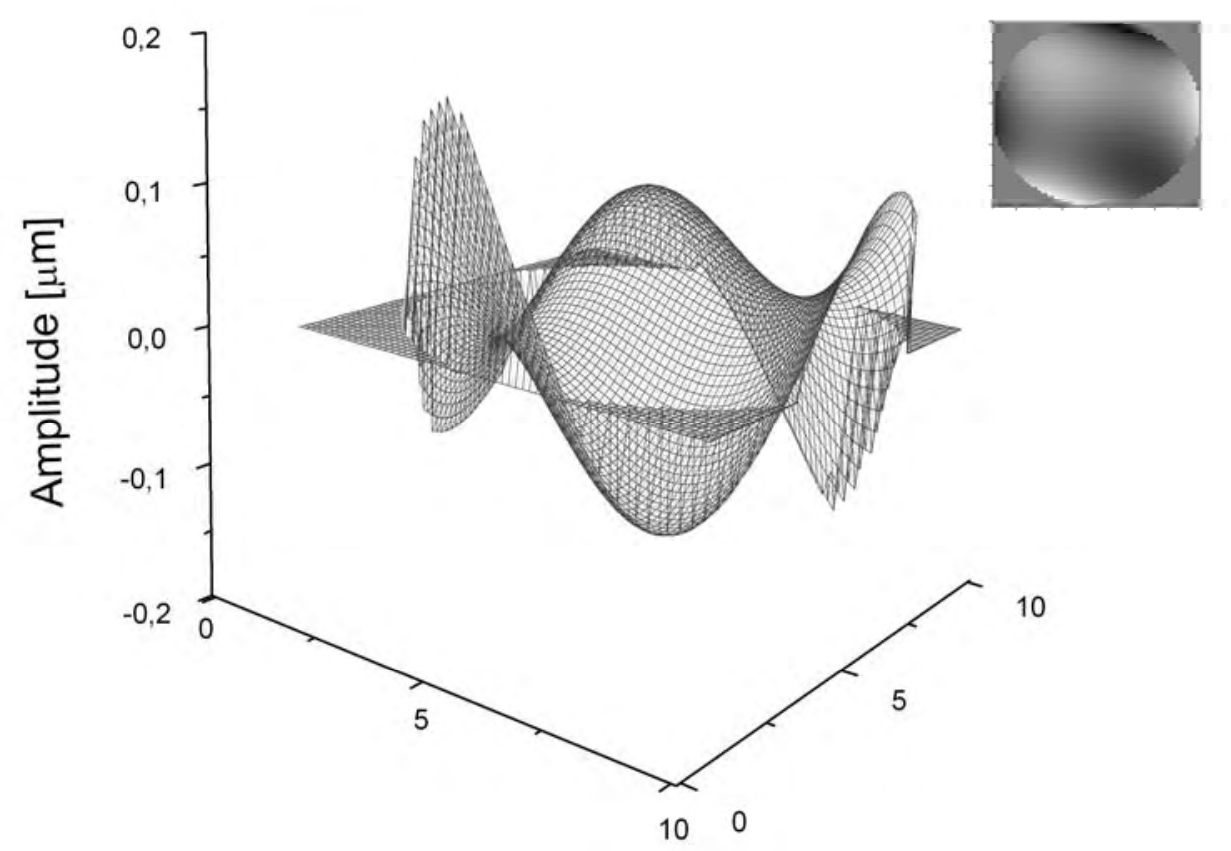

Figure 6: Profile of the mild aberration introduced, which contains mostly coma, astigmatism, trefoil and spherical aberration.

In practice, dynamic distortions of a given wavefront occur smoothly and the Adaline weights oscillate within a somewhat limited range. By modifying the wavefront only slightly ( $\lambda / 16 \mathrm{rms}$ ) we registered the algorithm convergence in a single epoch to mse $=0.0018$ (accuracy $\sim \lambda / 1000$ ), in $200 \mu \mathrm{s}$. Figure 7 shows the residual aberration introduced. 


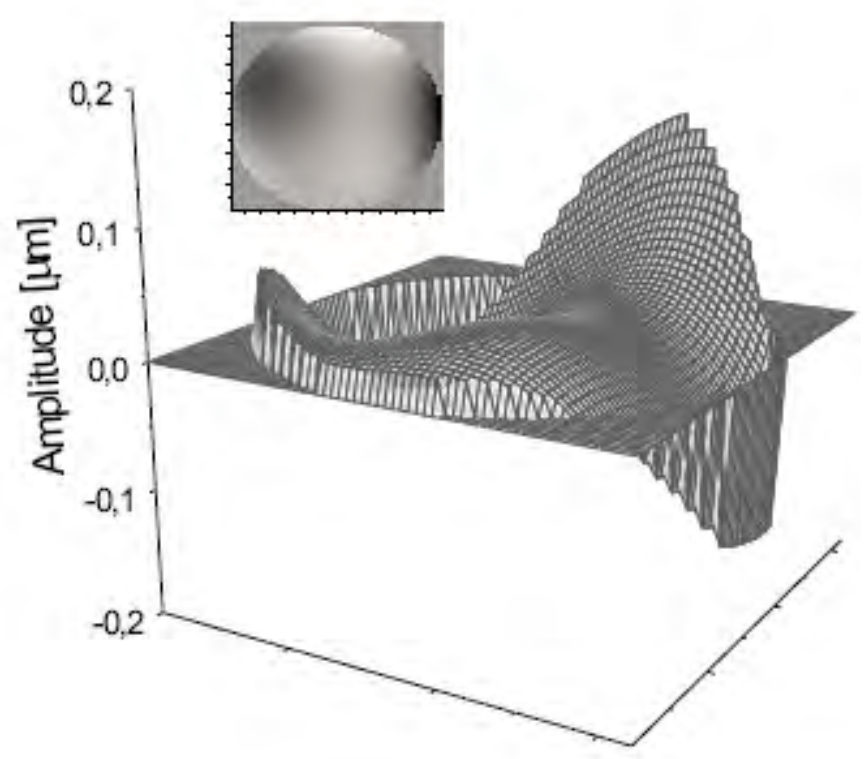

Figure 7: Residual aberration introduced to the system.

As the sensor has been read at $1 \mathrm{kHz}(1 \mathrm{~ms})$ and all software routines take less than $400 \mu \mathrm{s}$, the system has potential to be operated at rates higher than $600 \sim \mathrm{Hz}$.

To test the effect of one iteration of the AO system, we aimed at partially compensating the aberration in Figure 6 by applying a voltage to the mirror proportional to the weights that correspond to $m s e=0.0018$. The resulting spots displacements are kept within $\sim 10 \%$ of the reference grid, which corresponds to approximately $\lambda / 5$. This indicates that a single iteration already complies with the wavefront accuracy of $\lambda / 4$ usually used as a rough estimate of quality in conventional optical shop tests (5). The rate of accuracy improvement with increasing iterations is yet to be investigated. To improve the accuracy we need to employ a more accurate reference wavefront (6) and a larger number of quadcells, which might lower the system operational frequency.

\section{CONCLUSIONS}

The use of a simple Adaline neural method for the reconstruction of a wavefront based on the optical sensor signals proves effective and renders an error negligible compared to the system errors. It is rather immune to round-off errors and handles aliasing acceptably. An adaptive mirror can be controlled more directly from the sensor signals through a slight modification to the neural algorithm. A single iteration of the adaptive optical system, fed by coefficients delivered by the neural net, yielded an $r m s$ wavefront divergence from reference better than $\lambda / 4$. These results indicate that further iterations are bound to improve accuracy even more. The algorithm is suitable for real-time system operation, requires no extensive training and is receptive to dynamic adaptability of the input functions. 


\section{ACKNOWLEDGMENTS}

This project has been supported by FAPEMIG (Fundação de Amparo à Pesquisa do Estado de Minas Gerais), and by the Dutch Technical Foundation (STW). The authors thank the Electronic Instrumentation Lab. and DIMES (TU-Delft/The Netherlands); and in particular Dr.Mikhail Loktev (OKO Technologies) for his prompt assistance in technical matters.

\section{REFERENCES}

1. B. Widrow and M.E. Hoff, Jr., "Adaptive switching circuits.” Western Electric Show and Convetion Record Part 4, p.96-104, 1960

2. M. Born, E. Wolf, "Principles of Optics,” Pergamon Press, Oxford, 6th edition, 1986.

3. D. W. de Lima Monteiro, T. Nirmaier, G. Vdovin, A. Theuwissen., "High-speed wavefront sensor compatible with standard CMOS technology," Sensors and Acutators A, 109 (3), p.22-230, 2004

4. G. Vdovin, “Adaptive mirror micromachined in silicon,” Delft University Press, Delft, 1996

5. D. Malacara, “Optical Shop Testing,” $2^{\text {nd }}$ ed., John Wiley and Sons Inc., New York, 1992

6. G. Vdovin, "Optimization-based operation of micromachined deformable mirrors," Proc. SPIE, 3353, p.902-909, 1998 